\title{
CHANNELING PROJECTS AT LNF: FROM CRYSTAL UNDULATORS TO CAPILLARY WAVEGUIDES
}

\author{
S.B. Dabagov, INFN Laboratori Nazionali di Frascati, I-00044 Frascati (RM), Italy
}

\section{Abstract}

Since the middle of the last century, Frascati National Laboratories (LNF INFN) are well known in the world for pioneering research in the particle interaction and synchrotron radiation physics fields. Good experience in designing accelerators, storage rings and beamlines for synchrotron radiation allows presently to the LNF to be in the frontier for the construction of new X-ray generation sources.

This report is an introduction to new research activity "Coherent Scattering Phenomena for Radiations in Solids" started in Frascati within the approved projects SPARC / SPARX / PLASMON-X. The main idea of the projects are developing research area to study the channeling phenomena for charged and neutral particles in periodic solid structures.

The "Channeling 2004" workshop organized by LNF in November 2004, has shown a strong interest in the international collaboration, and the Advisory Committee of the meeting together with the INFN LNF administration have decided to organize an international conference "Channeling 2006" in June 2006.

\section{INTRODUCTION}

Interaction of different kinds of radiation with matter has been studied since scientific community started to operate with penetrating radiations. Among various research directions, the coherent phenomena of the radiation passage through solids, as coherent bremsstrahlung, channeling and related effects in crystals, attract attention because it can create a strong flux of photons in broad energy intervals, starting from optical frequencies, via soft and hard X-ray, up to $\gamma$-ray frequencies. The latter makes this research field more and more attractive due to the importance of creating new intensive photon sources like the next generations of synchrotrons, free-electron lasers, Z-pinch machines, etc. Another approach to the problem of searching for intensive radiation sources is to increase the radiation density of existing X-ray and $\gamma$-sources that can be solved by means of novel optical elements, for instance capillary optics. Passage of charged particles through periodic systems and propagation of neutral particles (photons, neutrons) in capillary systems of micron or submicron channel sizes, even strongly different by nature, have much in common, as both can be described within the frame of channeling theory, surface or bulk. Therefore, the use of this analogy can significantly simplify researches in both areas.

Many intense X-ray sources are now available for scientific and technological applications. The recent ideas and the continuous developments bring X-ray sources, optics and detectors to performances that were almost unbelievable a few years ago. The new generation of powerful table-top devices for elemental and diffraction analysis, but in particular the many synchrotron radiation facilities all around the world that deliver bright and ultrabright X-ray beams to hundreds of users investigating with different techniques the atomic structure of matter witness the almost exponential increase of X-ray brilliance. X-ray sources produced countless discoveries, ranging from atomic scale maps of proteins to structures of hightemperature superconducting materials. However, only now for the first time in decades, thanks also to the developments of new optical elements such as refractive optics, multilayers, polycapillary lenses, phase gratings, etc., $\mathrm{X}$-ray scientists look at a new scenario that promise to be more exciting than ever. Researchers and funding agencies in Europe, USA and Japan are supporting the construction of new X-ray sources capable not only to generate the most brilliant sources but also to produce short $\mathrm{X}$ ray pulses comparable with the dynamics of the chemical bonds. Actually, this class of machines, called free electron lasers (FELs), will be able to deliver powerful beams of coherent X-rays probably reshaping the landscape of Xray science.

Since the middle of the last century Frascati's National Laboratories are well known in the world for pioneering research in the particle interaction and coherent radiation physics fields. Good experience in designing accelerators, storage rings and beamlines for synchrotron radiation allows presently the LNF to be in the frontier for the construction of new X-ray generation sources, as FEL, Thomson scattering, coherent bremsstrahlung, channeling radiation, etc. In order to discuss new results, exchange experiences and ideas by various groups in the world, form the basis for future research activities within new collaborations, the International workshop "Channeling 2004" was held in Frascati (Rome) on November 2-6, 2004.

\section{FIRST FRASCATI'S EXPERIMENTS}

Perhaps the first idea on the processes of radiation emission by charged particles traversing solids were published by Williams in 1934. Then Ferretti developed semi-qualitative theory of the phenomena based on the Weiszacker-Williams method of the virtual quanta [1]. After that work we are finding a number of papers by TerMikaelian, Purcell, Feinberg, Pomeranchuk, Dyson, and Überall devoted to theory of electromagnetic radiation by charged particles in matter (see [?, ?, 4] and Refs in). The full calculations of the cross sections and polarization 
within the Born approximation were first described in papers $[5,6]$.

The obtained theoretical results stimulated the performance of experiments by means of the high-energy electron accelerators. However, first experimental results were in apparent contradiction with the theoretical ones. Indeed the measurements $[7,8]$ showed an enhancement in the low region of the bremsstrahlung spectrum for a certain crystal orientation, while the theoretical expectation, was a minimum! This contradiction between theory and experimental results was due to the insufficient resolution of angle and energy measurements.

In 1959 Diambrini Pallazi proposed an experimental research program to be realized at the Frascati's $1 \mathrm{GeV}$ electrosynchrotron. The final purpose was to develop a source of a polarized photon beam useful for experiments in particle physics that were planned at the Frascati laboratories. The main problem to be solved was to obtain an energy and angular resolution higher than that used in the previous experiments [9].

The experiments at Frascati were first carried out by Bologna, Diambrini and Murtas, and later also by G. Barbiellini. These experiments in both the electron pair production and bremsstrahlung showed the expected central minimum. However, having a qualitative agreement with the Überall's results, a systematic difference in the results was also revealed $[10,11]$.

A further experimental investigation carried out with a better angular resolution showed an unexpected fine structure in the angular dependence of the bremsstrahlung cross sections. It was realized that the approximation of continuous lattice planes used in the previous calculations was unsuitable for bremsstrahlung in the $\mathrm{GeV}$ region, and new calculations, taking into account the actual structure of the lattice planes, are necessary. It was clear that also the bremsstrahlung spectrum would have large peaks with high polarization. Detailed calculations and precision measurements on the discovered coherent-spectrum bremsstrahlung and on the polarization have been carried out by the Frascati's group, finding good agreement between theory and experiment [12].

These authors also proposed a new method for measuring the polarization of very high energy photons by using a second crystal as analyzer $[13,14,15,16]$.

The experimental method was applied in almost all the electron accelerators around the world, for photoproduction experiment For example, the author was invited for explaining this new method in several labs, like DESY, Cornell University (USA), Harvard University (USA, MIT), Yerevan electro synchrotron lab (Armenia), Tokyo electrosynchrotron lab (Japan) [17, 18, 19, 20].

\section{SPARC/SPARX/PLASMON-X}

The overall SPARC (Sorgente Pulsata Auto-amplificata di Radiazione Coerente) [21] project consists of 4 main lines of activity aiming at several goals: their common denominator is to explore the scientific and technological issues that set up the most crucial challenges on the way to the realization of a SASE-FEL based X-ray source, the SPARX proposal [22] ((Fig. 1)).

These are:

- $150 \mathrm{MeV}$ Advanced Photo-Injector;

- SASE-FEL Visible-VUV Experiment;

- X-ray Optics/Monochromators;

- Soft X-ray table-top Source.

Since the performances of X-ray SASE-FEL's are critically dependent on the peak brightness of the electron beam delivered at the undulator entrance, investigations have been performing for two main issues - generation of the electron beam and bunch compression via magnetic and/or RF velocity bunching - by means of an advanced system delivering $150 \mathrm{MeV}$ electrons, the minimum energy to avoid further emittance dilutions due to the timedependent space charge effects.

In order to study the problems related to matching the beam into an undulator and keeping it well aligned to the radiation beam, as well as the generation of non-linear coherent higher harmonics, a SASE FEL experiment with the $150 \mathrm{MeV}$ beam, using a segmented undulator with additional strong focusing, will be organized to observe FEL radiation at $530 \mathrm{~nm}$ and below. The X-ray FEL radiation will provide unique radiation beams to users in terms of peak brightness and pulse time duration (100 fs), posing at the same time severe challenges to the optics that is necessary to guide and handle such radiation. This project is pursuing also a vigorous $R \& D$ activity on the analysis of radiation-matter interactions in the spectral range typical of SASE X-ray FEL's (from 0.1 to $10 \mathrm{~nm}$ ), as well as the design of new optics and monochromators compatible with these beams. In order to test these optics and to start the R\&D on applications, the project undertakes an upgrade of the presently operated table-top source of X-rays at Politecnico Milano, delivering $10^{7}$ soft X-ray photons in $10-20 \mathrm{fs}$ pulses by means of high harmonic generation in a gas.

$\mathrm{X}$-rays are presently utilized in many research and application fields, for instance, in imaging technique and in the analysis of industrial products. The SPARX source will be able to boost into excellence the actual applications and to steer research and application toward new objectives. There will be implications on many disciplines and innovative technologies will be developed in the field of material science, biology, medicine, non linear optics, $\mathrm{x}$-ray microscopy; new methods will be developed in protein cristallography.

SPARX source ability to deliver an enormous amount of energy on small areas will make possible to study effects that, up to now, can only be theoretically predicted. In particular interesting results are expected in the field of photochemistry, with important industrial applications. High peak brightness and short pulse duration (a few fs) will be the main characteristics of the SPARX source. By using a $2.5 \mathrm{GeV}$ linear electron accelerator and two magnetic undulators it is possible to obtain radiation at $10 \mathrm{~nm}$ and 1.5 


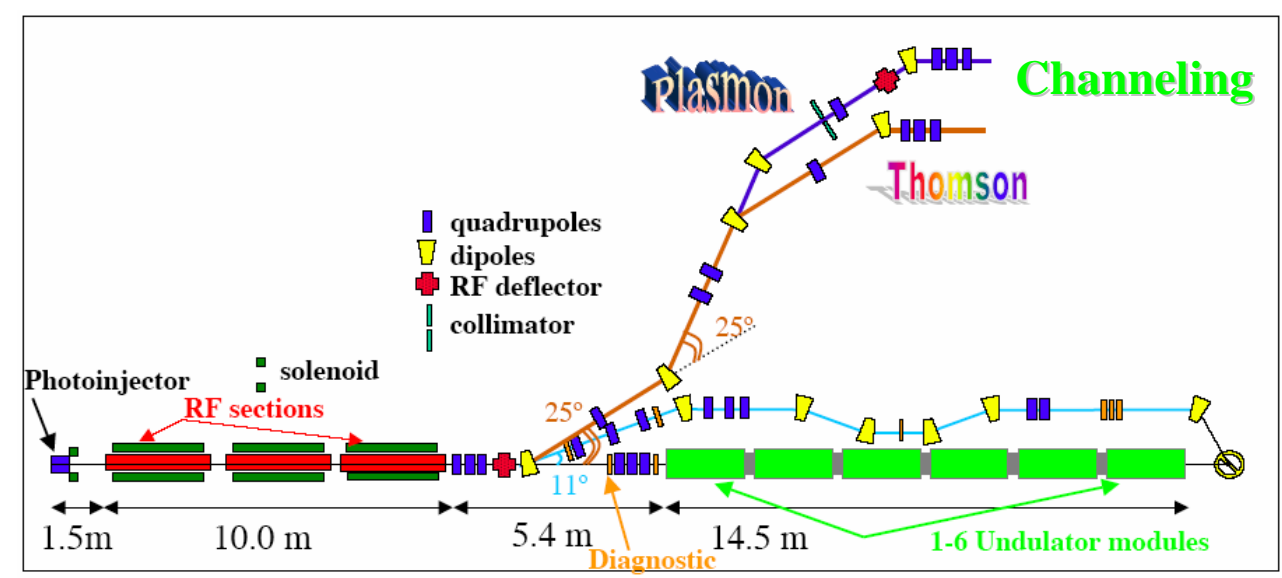

Figure 1: Schemes of different experimental layout disposition.

$\mathrm{nm}$. Exploitation of 3rd and 5th harmonics will allow emission in the range between 10 and $2 \mathrm{~nm}$ for the first beam line and between 1.5 and $0.3 \mathrm{~nm}$ for the second beam line.

The aim of the PLASMON-X project [23] is to provide the LNF with a world-class, high-power laser facility suitable for the development of an innovative, highgradient acceleration technique based upon super-intense and ultra-short laser pulses, and $\mathrm{X} / \gamma$-ray tunable sources using Thomson scattering of optical photons by energetic electrons. The facility will be built in close interaction with the SPARC project presently in progress.

The main purpose of the facility will consist in R\&D activity aimed at the following objectives:

1. demonstration of high-gradient acceleration of electrons injected into electron plasma waves excited by ultrashort, high-power laser pulses;

2. development of a monochromatic and tunable X-ray source in the 20-1000 keV range, based upon Thomson scattering of laser pulses by relativistic electrons.

Both lines require very bright and short electron bursts together with very high power laser pulses of sub-100 fs duration. Studies at the forefront of research in this field consistently indicate that the power level and the focused intensity for a laser system appropriate for these studies are $>10 \mathrm{TW}$ and $>10^{19} \mathrm{~W} / \mathrm{cm}^{2}$, respectively.

The activity will be carried jointly by the INFN and CNR laboratories.

The R\&D activity carried out at this facility will be open to some extent to external users, in particular for what concerns bio-medical oriented uses of the available sources for diagnose and therapy.

\section{COMPARISON OF SPECTRAL CHARACTERISTICS FOR CHANNELING RADIATION, BREMSSTRAHLUNG AND THOMSON SCATTERING}

Similar to bremsstrahlung (B) (brake radiation, BetheHeitler radiation) channeling radiation from relativistic electrons in crystals $(\mathrm{ChR})$ has an angular divergence of about $\Delta \vartheta \simeq \gamma^{-1}$, where $\gamma=\frac{E}{m_{0} c^{2}}$ is a Lorentz-factor, $E$ is an electron energy. However, typical crystal thickness to generate these types of radiation varies due to the fact that there is big difference in the processes of multiple scattering. The latter results in strong enhancement for spectral and angular characteristics of $\mathrm{ChR}$ in comparison to $\mathrm{B}$ for $\gamma \gtrsim 10^{3}$ (see in [24],nim98,khoca-nim98,shul'ga).

Let consider the expressions for angular densities of $\mathrm{B}$. A maximum in the angular density of $\mathbf{B}(d W / d \vartheta)_{B}$ is limited by the multiple scattering of electron in a matter. A characteristic thickness $x_{B}$ at radiation within the angles $\gamma^{-1}$ is defined by the expression for averaged angle of scattering (with logarithmic accuracy)

$$
\frac{1}{\gamma^{2}}=\left(\frac{E_{s}^{2}}{E^{2}}\right)\left(\frac{x_{B}}{R}\right)
$$

where $R$ is a radiation length, $E_{s}=21 \mathrm{MeV}$. So we have a simple formula for the estimations

$$
x_{B}=\frac{R}{1764}
$$

from which it is easy to obtain $x_{B}=56 \mu \mathrm{m}$ for $\mathrm{Si}, 1.7 \mu \mathrm{m}$ for $\mathrm{W}$, and $73 \mu \mathrm{m}$.

Then the energy emitted within the angles $\gamma^{-1}$ can be estimated as

$$
\Delta E=\left(\frac{d E}{d x}\right) \Delta x=\left(\frac{E}{R}\right) x_{B}=\frac{E}{1764}
$$

Hence approximately for $\Delta \vartheta \simeq \pi \gamma^{-2}$ one writes

$$
\Delta E=\left(\frac{d W}{d \vartheta}\right)_{B} \Delta \vartheta=\frac{E}{1764}
$$

In the same way one can consider an oriented crystal, however we need additional information on energetic lost for the spontaneous radiation. The dependence of energy lost for the spontaneous radiation has a form of $d E / d x \sim$ 
$E^{2}$. Now a characteristic length, on which the radiation happens in oriented crystal, is determined by a dechanneling length $x_{C h}$. The emission of energy into the angles of $\gamma^{-1}$ for the main harmonics, i.e. for the narrow spectral frequency range, takes place up to an optimal value of energy that is defined by the dipole character of radiation. At the planar channeling $E_{\text {opt }}$ is close to $5-10 \mathrm{GeV}$, and at the axial channeling is about $1 \mathrm{GeV}$.

Let introduce a ratio of the angular densities of radiation by the expression

$$
\eta_{1}=\frac{(d W / d \vartheta)_{C h}}{(d W / d \vartheta)_{B}}
$$

Because of the angular fans, inside of which B and ChR concentrate, are equal, we obtain

$$
\eta_{1} \simeq \frac{(d E / d x)_{C h}}{E / R} \frac{x_{C h}}{x_{B}},
$$

where $x_{C h}=\int \chi(x) d x ; \chi(x)$ is a portion of the charged particles beam remained in the mode of channeling at the crystal thickness of $x$. For example, the dechanneling lengths for positrons $\left(E_{e+}=200 \mathrm{MeV}\right)$ approximately equal to $50 \mu \mathrm{m}$ for a monocrystal $\mathrm{Si}(110)$, and $30 \mu \mathrm{m}$ for diamond. The close values are obtained also for the case of planar positron channeling in $\mathrm{W}$ crystals (for positrons $x_{C h} \approx x_{1 / 2}$ is a depth, at which a half of the particles captured by the crystal channels dechannels). For the axial channeling of positrons the dechanneling length increases by about one order of value. For electrons at the axial channeling in W we obtain $x_{1 / 2} \approx 10 \mu \mathrm{m}$ at $E=200 \mathrm{MeV}$ (close values have been obtained also for $\mathrm{Si}$ ). However, we have to underline that for the large depths (more than $x_{1 / 2}$ ) the rate of dechanneling essentially decreases. Hence there is an opinion to use the crystals of larger thickness than $x_{1 / 2}$. From the Eq.(6) it follows that at $E=200 \mathrm{MeV}$ $\eta_{1} \simeq 10 \div 10^{2}$.

Taken the problem of the spectral-angular characteristics of radiation, i.e.

$$
\eta_{2}=\frac{\left(d^{2} W /(d \vartheta d \omega)\right)_{C h}}{\left(d^{2} W /(d \vartheta d \omega)\right)_{B}}
$$

we will calculate the thickness, at which the ratio $\eta_{2}$ has a maximum. Approximately one can be written

$$
\eta_{2}=K_{1} K_{2}
$$

where $K_{1}=\frac{(d W / d \omega)_{C h}}{(d W / d \omega)_{B}}$ describes a ratio of spectral densities obtained experimentally; $K_{2}=\frac{x_{C h}}{x_{B}}$. It is well known that the dechanneling length is proportional to energy of particle: $x_{C h} \sim E$. Thus, if $K_{1}$ is a constant or changes a little, then $\eta_{2}$ increases with the growth of particle energy. As analysis shows, one expects a factor of $\eta_{2} \approx 100 \div 300$ at $E \leq 1 \mathrm{GeV}$.

Comparison for the Eqs.(6) and (7) speaks on the fact that the main features (advantages) of channeling radiation can be displayed studying the spectral and angular characteristics of radiation. Due to the fact that for the energy range $E=0.1 \div 0.5 \mathrm{GeV}$ spontaneous radiation can be described in the dipole approximation we can conclude that the maximum radiated energy will be obtained for the maximal energy of channeled particles. Additionally to this fact we have to note that the crystal thickness should be optimized to be equal or more than the dechanneling length.

The study of spectral and angular distributions of $\mathrm{ChR}$ shows a possibility for creating a powerful source of narrow forwarded electromagnetic radiation. A number of emitted photons is evaluated by the following

$$
N_{C h} \simeq \frac{1}{137} \frac{x_{C h}}{\lambda_{C h}} K,
$$

where $K=\gamma^{2} \psi^{2}, \psi$ is a critical angle of channeling (the Lindhard's angle), for $E=0.1 \div 10 \mathrm{GeV} K \approx 1, \lambda_{C h} \simeq$ $d / \psi, d$ is a channel's width.

In the case of bremsstrahlung we can estimate a number of photons as

$$
N_{B} \simeq \frac{x_{B}}{R} \ln \frac{\omega_{2}}{\omega_{1}}
$$

From the examination of two last relations we see that one channeled particle enables to emit photons, the number of which in one or two orders exceeds one for B. Herewith the angular density of $\mathrm{ChR}$ exceeds in more than two orders the angular density of $\mathrm{B}$.

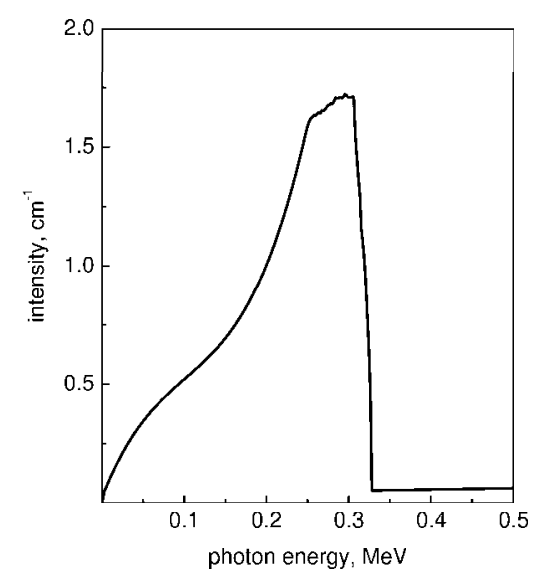

Figure 2: Intensity of ChR for relativistic positrons $E_{e+}=$ $200 \mathrm{MeV}$ in Si (110)/Maisheev/.

In Fig. 2 one presents the dependence of ChR intensity versus emitted photon energy within the angles of $\gamma^{-1}$ that was obtained by the simulation of planar channeling of positrons with energy $E_{e+}=200 \mathrm{MeV}$ in $\mathrm{Si}$ (110). As seen a channeled positron can produce in the maximum more than one photon of about $300 \mathrm{keV}$ per $\mathrm{cm}$ that exceeds at least in one order the number of bremsstrahlung photons.

There is another type of radiation that have to be considered as a competitor of $\mathrm{ChR}$ too, namely, coherent 
bremsstrahlung (CB). A braking particle radiated coherently in crystals, is scattered as typical particle, in other words, for this event there is no a suppression of scattering as we observe in the case of channeling. That's why a factor of $x_{C h} / \lambda_{C h}$ responsible for the sharp increase in the spectral-angular density of channeling radiation, is absent for CB. For the channeled particles the radiation concentrates in the narrower spectral range, for which its density is essentially higher than for CB. Moreover, due to the suppression of multiple scattering, $\mathrm{ChR}$ is more forwarded than $\mathrm{CB}$, and at last, an integral of $\mathrm{ChR}$ over the given range of frequencies and angles is significantly larger than coherent one.

This discrepancy can be explained by the various mechanisms of radiation: ChR regards to particle deflection by the averaged field of atomic rows and planes, while CB to periodical collisions of particles with the atomic rows and planes that does not cause a meaningful deflection of particle from the quasi straight trajectory. Hence, they are characterized by various radiation frequencies and observed typically for the different crystal-beam orientations: pure $\mathrm{CB}$ is detected for the entrance angles that overcome the critical angles of channeling. However, for some specific cases of a beam transversing a crystal under the small angles both along the plane and the row, there is a possibility to observe simultaneously channeling and coherent radiation ( or combination) but for the different frequency ranges.

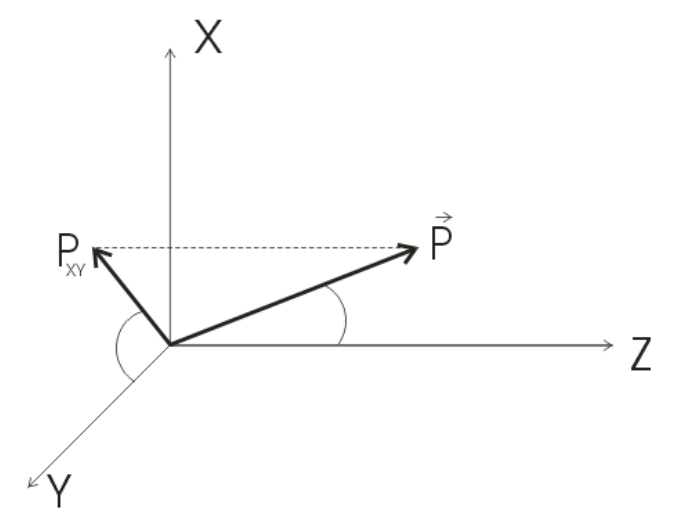

Figure 3: Coordinate system for a projectile in crystal.

Below we present a few graphs of spectral distributions of electromagnetic radiation by relativistic electrons and positrons in oriented crystals. The graphs for CB calculation and simulation are shown by dott-dashed line and thick line, correspondingly ; dashed line - for Bethe-Heitler radiation spectrum; $\alpha$ is the angle between the projection $p_{x y}$ of the particle momentum on the (x,y) plane and y-axis, $\psi_{c}$ is the axial channeling angle, $\psi$ is the angle between particle momentum $\vec{p}$ and crystal axis (z-axis) (see Fig.3).

As seen from the Figs.4, 5, in comparison to B we have enhance in the emission of photons for classical CB calculation as well as for the simulation. However, the results

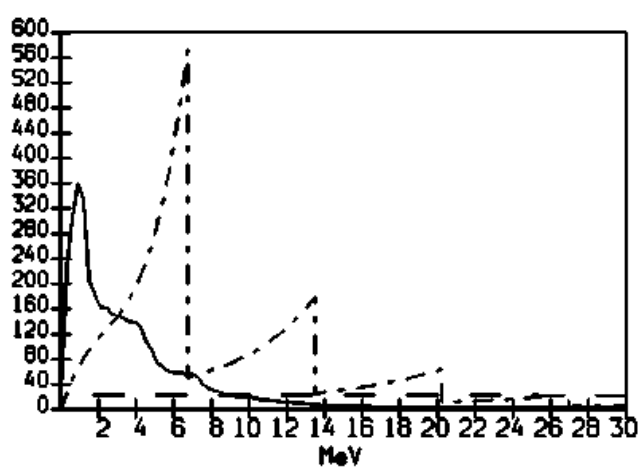

Figure 4: Spectrum of coherent radiation of positrons with energy $E_{e+}=0.5 \mathrm{GeV}$, moving in silicon crystal at small angles to $i 100_{i}$ axis $\left(\psi=\psi_{c}, \alpha=0\right) /$ Shul'ga et al/.

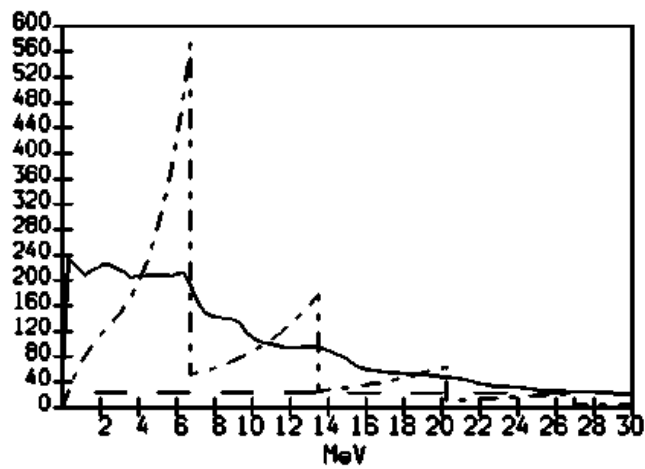

Figure 5: Spectrum of coherent radiation of positrons with energy $E_{e+}=0.5 \mathrm{GeV}$, moving in silicon crystal at small angles to $i 100_{i}$ axis $\left(\psi=\psi_{c}, \alpha=0\right) /$ Shul'ga et al/.

of simulations strongly varies from the calculations by the classical formulas. Taking into account the analysis above done, we expect to have another gain factor in the radiation exit at the particle channeling.

Now we consider another source of radiation, namely, Thomson scattering (TS), in comparison to ChR. Even these two types of radiation are similar by their description, we have to note that the mechanisms of photon production are different. ChR is based on the scattering of relativistic particles (electrons and positrons) in the field of averaged potentials of crystal rows or planes, while in the case of TS we deal with laser photons scattered by relativistic electrons. In both cases we have to keep in mind different factors that can suppress significantly the photon emission: multiple incoherent scattering by crystal atoms for the channeling and mutual refractions in a "photon-electron" system for the TS.

As well known photon energy emitted by relativistic channeled particle $(\gamma \gg 1)$ can be expressed by

$$
\omega_{l a b}^{C h R} \simeq \frac{2 \gamma^{2}}{1+\vartheta^{2} \gamma^{2}} \omega_{0}^{C h R}
$$

where $\omega_{l a b}$ is the observed energy of ChR, $\omega_{0}$ is the energy 
of particle oscillation that is defined by the interaction potential, i.e. crystal parameters, while in the case of TS the photon energy (radiated in the forward direction, $\vartheta \ll 1$ ) may vary between two extremes $(\vartheta=0$ and $\vartheta=\pi)$, thus

$$
\omega_{l a b}^{T S}\left\{\begin{array}{c}
\vartheta=0 \\
\vartheta=\pi / 2 \\
\vartheta=\pi
\end{array}\right\} \simeq\left\{\begin{array}{l}
1 \\
2 \\
4
\end{array}\right\} \frac{\gamma^{2}}{1+\vartheta^{2} \gamma^{2}} \omega_{0}^{T S}
$$

From this relations it follows that for these types of radiation we obtain the next dependences: $\omega_{\text {lab }}^{C h R} \propto \gamma^{3 / 2}$ and $\omega_{l a b}^{T S} \propto \gamma^{2}$. For the electron/positron energy of 100$200 \mathrm{MeV}$ the emitted radiation interval (frequencies) will be practically the same. So we have to compare total radiated powers for the both types of radiation. The number of photons emitted per unit of time at channeling increases with the increase of the electron/positron energy by the law $\propto \gamma^{1 / 2}$ whereas at TS this value is independent on the energy of projectile. However, in the same time the radiated power for the both cases $P \propto \gamma^{2}$. The fact that mathematical description for both ChR and TS is the same, allow us to introduce a simple comparison factor as

$$
f \simeq \frac{\mathbf{A}_{C h}^{2}}{\mathbf{A}_{T S}^{2}} \frac{L_{C h}}{L_{T S}}
$$

where $\mathbf{A}$ is the strength parameter of a field (crystal or laser) with the interaction length $L$ (in the case of channeling the latter is defined as an effective length of channeling, i.e. $L_{C h}(z) \simeq \int_{0}^{z} N_{c h}(z) d z$, is the integral of channeled particles at fixed depth, while for the TS $L_{T S}$ by the laser beam sizes and the mutual orientation). Analyzing the existing experimental data we can reduced the strength parameters for electron scattering in crystals

$$
\begin{array}{cccc} 
& \mathrm{Si}\langle 110\rangle & \mathrm{C}\langle 100\rangle & \mathrm{W}\langle 111\rangle \\
\mathbf{A}_{C h}^{2}, \mathrm{eV} / \AA^{3} & \sim 520 & \sim 580 & \sim 10000
\end{array}
$$

and $\mathbf{A}_{T S}^{2} \sim 700 \mathrm{eV} / \AA^{3}$ for the $10 \mathrm{TW}$ laser with a beam diameter of $0.1 \mathrm{~mm}$.

For 100-200 MeV electrons/positrons in the ideal situations when we have a very powerful laser beam, very high time and spatial synchronization, and without any destructive effects, the radiated power for the TS exceeds the power for channeling radiation. However, such a situation is very difficult to realize. If we consider more realistic case for the TS, namely, the laser power of $5 \mathrm{~kW}$ with a $0.1 \mathrm{~mm}$ beam diameter at wavelength of $1 \mu \mathrm{m}$, we can get $\sim 10^{-8} \mathrm{ph} / \mathrm{e}^{-}$at $L_{T S} \approx 1 \mathrm{~cm}$. However, the exit of photons, for instance, from $E_{e+}=200 \mathrm{MeV}$ positrons planar channeled in $\mathrm{Si}$ (110) of $z=0.1 \mathrm{~mm}$ thickness may reach $\sim 10^{-3} \mathrm{ph} / \mathrm{e}^{+}$(some experiments indicate $\sim 5 \cdot 10^{-3} \mathrm{ph} / \mathrm{e}^{-}$ at planar channeling of $350 \mathrm{MeV}$ electrons and $\sim 3 \cdot 10^{-2}$ $\mathrm{ph} / \mathrm{e}^{-}$at axial channeling in $170 \mu \mathrm{m} \mathrm{Si}$, and photon energy with maximum for $\omega_{\text {lab }}^{C h R} \sim 0.8 \mathrm{MeV}$ ). Going down by the photon energy to X-ray region we can mention an experimental results with $\sim 10^{-3} \mathrm{ph} / \mathrm{e}^{-}$emission by $E_{e-}=100$ $\mathrm{MeV}$ electrons channeled in $105 \mu \mathrm{m} \mathrm{Si}$ (110) (note that this value can be essentially increased by the use of thicker crystals).

Hence one should be emphasized that channeling radiation is really effective source of photons in very wide range of frequencies. In the X-ray interval of photon energies it will provide strong electromagnetic radiation higher than we get by bremsstrahlung, coherent bremsstrahlung and Thomson scattering. However, in the latter we have to underline that Thomson scattering radiation has a higher degree of monochromaticity and is free from incoherent background, which is always present at channeling.

\section{X-RAY CHANNELING}

Propagation of $\mathrm{x}$-ray and neutron through microcapillaries has been intensively investigated in the last 20 years $[28,29]$ due to the high transmission efficiency of the radiation by the internal hollow part of microcapillaries, but also, due to the possibility to characterize new optical elements capable to deflect or bend intense radiation beams. These researches shown, in addition to the relevance of the capillary optical systems, that capillaries represent a fine tool to investigate fundamental dispersion features of $\mathrm{x}$-ray radiation by a surface $[30,31,32,33]$.

In microcapillaries the sizes of channels for radiation propagation are much larger than the effective wavelength of the radiation and the process dispersion is described within the framework of surface (superficial) channeling. Capillary optics manufacturing technology allows us to reach extremely small channel diameters, actually, the last (fifth) generation of these optics achieved submicron channel diameters. The last reports show that capillary optical elements with an inner channel diameter of a few tens of nanometers can be obtained with the existing technologies [34].

\section{Channeling in microcapillaries: Surface chan- neling}

As it has been shown recently, the propagation of X-ray photons through capillary systems exhibits a rather complex character [35]. Not all features shown in the experiments can be explained within the geometrical (ray) optics approximation $[36,37,38]$. On the contrary, the application of wave optics methods allows us to describe in details the processes of radiation spreading into capillaries.

The propagation of $\mathrm{X}$ radiation through capillary systems is mainly defined by its interaction with the inner channel walls. In the ideal case, when the boundary between hollow capillary and walls represents a smooth edge, the beam is split in two components: the mirror-reflected and refracted ones. The latter appears sharply suppressed in the case of total external reflection. The characteristics of scattering inside capillary structures can be evaluated from the solution of a wave propagation equation. In the first order approximation, without taking into account the roughness correction $\Delta \varepsilon(\mathbf{r})=0(\Delta \varepsilon$ is the perturbation in dielec- 


\section{Down to bulk photon and neutron channeling}

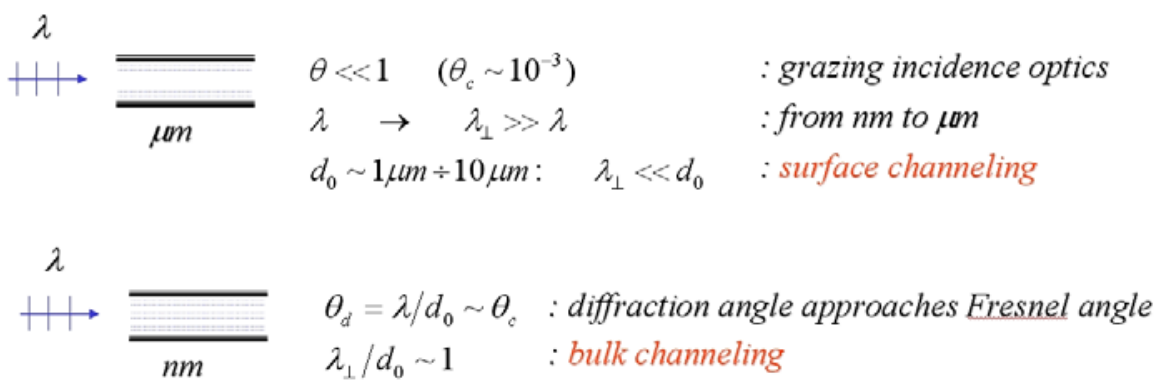

Figure 6: Changes in the propagation character of the radiation inside micro- and nanochannels.

tric permittivity induced by the presence of roughness), the wave equation in the transverse plane to the propagation direction reads

$$
\left(\nabla_{\perp}^{2}-k^{2} \delta\left(r_{\perp}\right)+k_{\perp}^{2}\right) E\left(r_{\perp}\right)=0
$$

where $E$ is a radiation field function, and $\mathbf{k} \equiv\left(\mathbf{k}_{\|}, k_{\perp}\right)$ is a wave vector.

Due to the fact that the transverse wave vector may be presented as $k_{\perp} \approx k \theta$ under the grazing incidence $(\theta \ll 1)$, an "effective interaction potential" is estimated by the expression

$$
\begin{aligned}
& V_{\text {eff }}\left(\mathbf{r}_{\perp}\right)=k^{2}\left(\delta\left(\mathbf{r}_{\perp}\right)-\theta^{2}\right)= \\
& =\left\{\begin{aligned}
-k^{2} \theta^{2} & , \quad r_{\perp}<r_{1} \\
k^{2}\left(\delta_{0}-\theta^{2}\right) & , \quad r_{\perp} \geq r_{1},
\end{aligned}\right.
\end{aligned}
$$

where $r_{1}$ corresponds to the reflecting wall position. From the latter the phenomenon of total external reflection at $V_{\text {eff }}=0$ follows, when $\theta \equiv \theta_{c} \simeq \sqrt{\delta_{0}}$ - the Fresnel's angle.
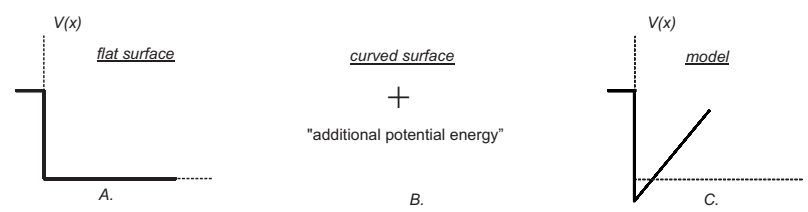

Figure 7: The change of the interaction potential between the flat surface (A.) and the curved one (B.). For simplicity, in calculations the "real potential" (B.) may be replaced by the model potential (C.).

When we introduce a curvature in the reflecting surface, the effective potential obtains an additional contribution. This term, that corresponds to the additional "potential energy", can be seen physically in the following way. Due to the reflecting surface curvature a photon receives an angular momentum $k r_{c u r v} \varphi$, where $r_{c u r v}$ is the curvature radius of the photon trajectory, $\varphi$ is an azimuthal angle in the cylindrical coordinates chosen along the direction of radiation propagation. The latter is supplied by the "centrifugal potential energy" $-2 k^{2} r_{\perp} /\left(r_{\text {curv }}\right)$

$$
V_{e f f}\left(\mathbf{r}_{\perp}\right)=k^{2}\left(\delta\left(\mathbf{r}_{\perp}\right)-\theta^{2}-2 \frac{r_{\perp}}{r_{\text {curv }}}\right) .
$$

The situation is explained in the scheme in Fig.7. Because of the variation in the spatial system parameters, the interaction potential has been changed from the step potential with the potential barrier of $k^{2} \delta_{0}$ to the well potential, with the depth and width defined by the channel characteristics.

In the following we briefly discuss a solution of the wave equation in the case of an ideal reflecting surface (i.e. without roughness), when the reflected beam is basically determined by the coherently scattered part of radiation $([39,40])$. The evaluation of the wave equation with the boundary conditions of a capillary channel shows that $\mathrm{X}$-radiation may be distributed over the bound state modes defined by the capillary channel potential (see below). It is important to underline here that the channel potential acts as an effective reflecting barrier, and then, an effective transmission of $\mathrm{X}$-radiation by the hollow capillary tubes is observed. While the main portion of radiation undergoes incoherent diffuse scattering [41], the remaining contribution (usually small) is due to coherent scattering that represents a special phenomenon, extremely interesting to observe and clarify [42].

Let us estimate the upper limit of curvature radius $\left(r_{\text {curv }}\right)_{\max }$ (which is defined by capillary/system of capillaries bending and/or by the radiation propagation trajectory), at which the wave behaviors are displayed under propagation of radiation in channels [30], by considering a photon with the wave vector $\mathbf{k}$ channeling into capillary with curvature radius $\left(r_{c u r v}\right)_{i}$ ( $i$-th trajectory). At small glancing angles, $\theta$, the change of the longitudinal (along the propagation direction) wave vector, $k_{\|}$, under reflection from the capillary wall is negligibly small; but mainly one changes the transverse wave vector, $k_{\perp}$,

$$
k_{\perp} \simeq k \theta\left(\theta<\theta_{c}\right)
$$

Correspondingly, from this relation it follows that the transverse wavelength will much exceed the longitudinal wave- 
length. This makes the interference effects observable even for very short wavelengths. Indeed,

$$
\lambda_{\perp}=\lambda / \theta>>\lambda
$$

quantum mechanical principles say that, in order to display the wave properties of a channeling photon, it is necessary that typical sizes of an "effective channel" $\delta_{i}$, in which waves have been propagating, be comparable to the transverse wavelength, i.e. $\delta_{i}(\theta) \simeq \lambda_{\perp}(\theta)$ (Fig.8). Taken into account the fact that the term for $\delta_{i}$ is defined by the $i$-th layer curvature radius $\left(r_{\text {curv }}\right)_{i}$ and the incidence angle $\theta$, namely, $\delta_{i} \simeq\left(r_{\text {curv }}\right)_{i} \theta^{2} / 2$, this condition may be rewritten in the following form:

$$
\left(r_{\text {curv }}\right)_{i} \theta^{3} \sim \lambda,
$$

from which we obtain $\left(r_{\text {curv }}\right)_{\max } \sim 10 \mathrm{~cm}$ for a photon of $\lambda \sim 1 \AA$ wavelength at glancing angle $\theta \simeq \theta_{c} / 3$. So, from this simple estimate we can conclude that the relation (18) provides a specific dependence for surface bound state propagation of X-rays - surface channeling - along the curved surfaces (for instance, in $\mu$-capillary systems) (see also [43]).

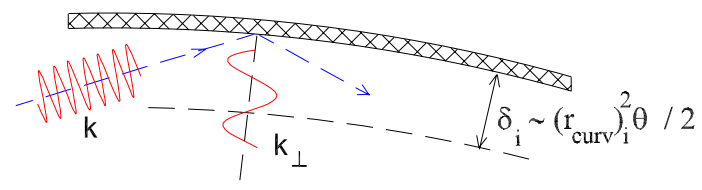

Figure 8: Illustration of X-ray reflection from the inner capillary surface. At glancing angles $\theta$, when the cross size of a beam $\delta_{i}(\theta)$ becomes comparable with the transverse wavelength $\lambda_{\perp}(\theta)$, the radiation is grasped in a mode of surface channeling.

Since the waveguide is a hollow cylindrical tube, if the absorption is considered to be negligible, the interaction potential, in which a wave propagates, is determined by Eq.(15) with the radiation polarizability parameter $\delta_{0} \simeq$ $\theta_{c}^{2}$. Solving the wave equation in cylindrical coordinates $\mathbf{r} \equiv\left(r_{\perp}, \varphi, z\right)$ we are mainly interested in the surface propagation, $r_{\perp} \simeq r_{1}$, which, in fact, defines a wave guiding character inside the channel of the curvature radius $r_{1}$ $\left(r_{\perp}=r_{1}-\rho,|\rho| \ll r_{1}\right.$, along the trajectory for the given mode $m$ )

$$
\begin{gathered}
E_{n} \simeq \sum_{m} C_{m} u_{m}(\rho) e^{i\left(k_{\|} z+n \varphi\right)}, \\
u_{m}(\rho) \propto\left\{\begin{array}{ccc}
A i\left(\rho+t_{m}\right) & , \quad \rho>0 \\
\alpha A i^{\prime}\left(t_{m}\right) e^{\alpha \rho} & , \quad \rho<0
\end{array} \quad(\alpha>0)\right.
\end{gathered}
$$

where $A i(t)$ is the Airy function, satisfying an equation $A i^{\prime}(t)=t A i(t)$ and decaying exponentially at $t>0$, $t_{m}$ is the $m$-th pole of the Airy function, and $\alpha$ is an inverse length characteristic of the capillary substance (the method used was described in detail in previous publications, see, for example, Ref. [44]). Evidently, these expressions are valid only for the lower-order modes and in the vicinity of a channel surface. The expression (19) characterizes the waves that propagate close to the waveguide wall, or in other words, the equation describes the grazing modal structure of the electromagnetic field inside a capillary (surface bound X-ray channeling states) (Fig.9).

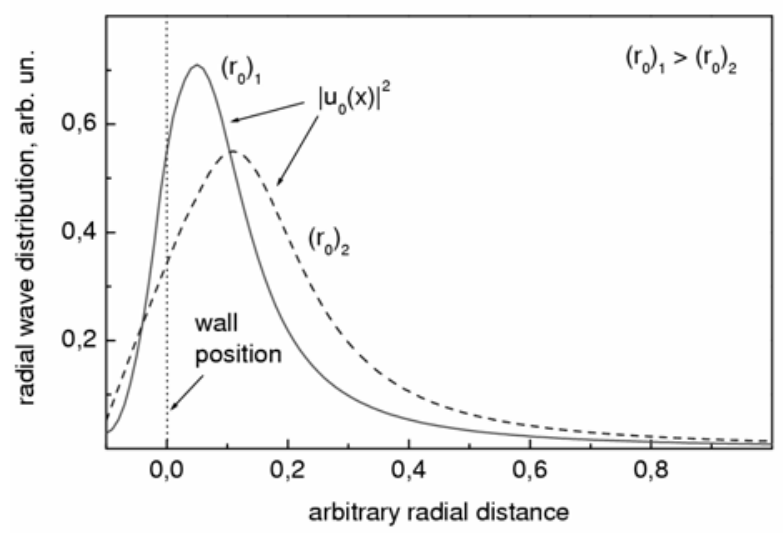

Figure 9: The radial distributions of the main bound mode of radiation inside a capillary channel for various channel diameters. The decrease of diameter $\left(2 r_{0}\right)$ results in a spatial displacement of the distribution away from the channel wall towards the center. The wall surface position is shown by the dotted line.

The solution shows also that the wave functions are exponentially damped both inside the channel wall: $\rho<0$ (as $e^{-2|\alpha||\rho|}$ ), and going from the wall towards the center: $\rho>0$ (as the Airy function decays). It should be underlined here that the bound modal propagation in the hollow part of the system takes place without the wave front distortion. The analysis of these expressions allows us also to conclude that almost all radiation power is concentrated in the hollow region and, as a consequence, a small attenuation along the waveguide walls is observed.

As for the supported modes of the electromagnetic field, estimating a characteristic radial size of the main grazing mode $(m=0)$ results in

$$
\bar{u}_{0} \simeq 0.75\left(\frac{\lambda^{2} r_{1}}{\pi^{2}}\right)^{1 / 3}
$$

and we can conclude that the typical radial size $\bar{u}_{0}$ may significantly overcome the wavelength $\lambda$, whereas the curvature radius $r_{1}$ in the trajectory plane exceeds the inner channel radius, $r_{0}: \bar{u}_{0} \gg \lambda$. For example, at the radiation wavelength $\lambda=10 \AA$ an estimation of the characteristic size $\bar{u}_{0}$ in the extreme case, when $r_{1} \simeq r_{0}$, gives us that $\bar{u}_{0} \gtrsim 75 \AA$ for a capillary channel with the radius $r_{0}=10 \mu \mathrm{m}$. 


\section{Channeling in nanocapillaries: Bulk channeling}

Simultaneously, design and test of new natural systems composed by many channels capable to guide radiation continued since the mid 80's. The first manuscript on the new crystalline structures [45] presently well-known as carbon nanotubes was published more than ten years ago [46]. Just from the beginning films of oriented carbon nanotubes represented a field of interest for the development of new electronic emitters, nanodevices, etc. [47]. The discovery of carbon nanotubes suggested also their use as collimators for hard $\mathrm{x}$-ray radiation and charged particles beams or for channeling of charged particles, $\mathrm{x}$-rays and neutrons [48, 49, 50, 51]. Features of nanotube morphology, e.g., the presence of inner cavity, address the possibility to achieve an efficient transmission of x-ray, thermal neutron and charged particle beams. Actually, nanotubes may be considered as nanoscale capillaries. However, for carbon nanotubes, the effective wavelength of $\mathrm{x}$-radiation becomes comparable with the diameter of channels and the description of the propagation as a reflection of photons from the internal surfaces of the tube walls is not consistent. Hence, instead of superficial effects of radiation scattering we deal with bulk features of radiation interaction with the system [52].

To understand the propagation nature and the scattering of soft x-rays inside channels of extremely small diameters is a nontrivial problem. The present investigations is probably the first experimental evidence of the influence of the nanotube alignment on the angular dependence of $x$-ray fluorescence of carbon.

As mentioned above, a nanotube can be considered as a capillary of very small inner diameter and wall thickness. However, there is a strong difference between a typical glass capillary and a nanotube from the point of view of radiation propagation inside these structures. First of all, the dielectric parameter as a function of the distance from the center of glass microcapillary channel varies with a step-law from zero in the inner hollow cavity to a constant value defined by the substance, for the channel wall. On the contrary, for a nanotube channel we observe a continuous change of the dielectric parameter value. However, the main difference is associated to the large difference in channel diameters that makes different the propagation character of the radiation inside microcapillaries and nanotubes. Indeed, radiation transmission by a cavity is defined as the ratio between the transverse wavelength of the radiation and the channel diameter $\lambda_{\perp} / d$. When this ratio is quite small, $\lambda_{\perp} / d \ll 1$, we can neglect wave features in the propagation process in the framework of the ray optics approximation. The same approach is not valid if $\lambda_{\perp} / d \simeq 1$ e.g., when the diffraction angle becomes comparable to the Fresnel angle $\theta_{c}=\frac{\omega_{0}}{\omega}$ (the critical angle of total external reflection), where $\omega_{0}=\left(\frac{4 \pi e^{2}}{m_{0}} N_{e}\right)^{1 / 2}$ is a plasmon energy, $N_{e}, e$ and $m_{0}$ are the electron density of matter, charge and mass of electron, respectively, $\omega$ is an energy quantum of radiation. This last condition occurs in nanosize channels and as a consequence nanochannels behave as radiation waveguides as demonstrated in Fig.6

The main characteristic of the propagation of radiation is correlated to the electron density $N_{e}(\overrightarrow{\mathbf{r}})$, which is position dependent. We may determine the density using an atomic potential approximations, the most simple of which is a Molier approximation. The use of a typical procedure for averaging interaction potential results in the following expression for a bent nanotube:

$$
\left\langle N_{e}(r)\right\rangle \approx \frac{r_{c u r v} N_{a} Z}{\pi a^{2}} \sum_{i} \alpha_{i} \beta_{i}^{2} \int_{0}^{\pi} d \theta K_{0}\left(\frac{\beta_{i} \rho}{a}\right),
$$

where $\rho^{2}=r^{2}+r_{\text {curv }}^{2}-2 r r_{\text {curv }} \cos \theta, r_{\text {curv }}$ is the radius of nanotube bending, $N_{a}$ is the atomic density, $a$ is the screening radius of carbon atomic form-factor, and $Z=6$. Evaluation of this relation gives estimation for the plasmon energy dependence presented in Fig.10.

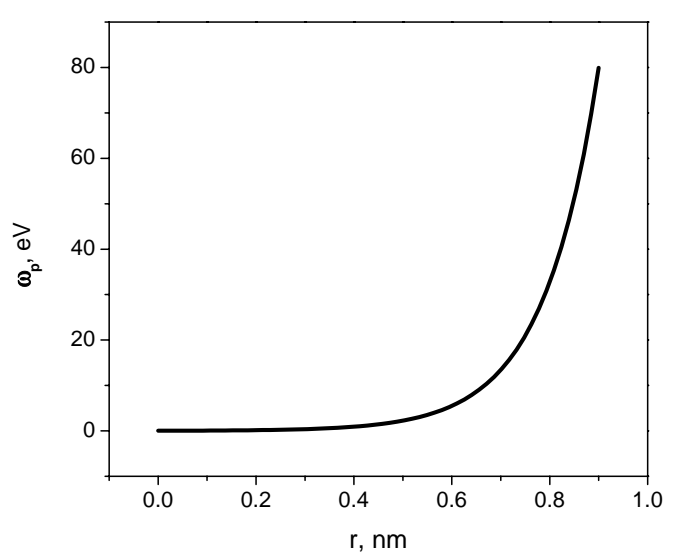

Figure 10: Plasmon energy distribution in the cross section of a nanotube

As expected, the behaviour has a deep maximum in the wall, while is a smooth continuous function crossing a nanotube cavity. Propagation of polychromatic radiation through a straight nanotube is accompanied by a continuous energy filter: the effective width of nanotube channel as wider as higher photon energy.

As follows from the analysis of the main parametes that defines the propagation characteristics of the radiation through a nanotube, the transverse wavelength $\lambda_{\perp}$ of the radiation is independent by the projectile energy but is a function of the electron density of the nanotube. The density difference at the nanotube axis and inside the nanotube wall determines the transverse wavelength which is just a few $\mathrm{nm}$.

Additionally to radiation channeling, the tunneling probability through the wall $\left(\propto \exp \left(-\frac{\Delta d}{\lambda_{\perp}}\right)\right)$ is quite high for a single wall nanotube due to the small thickness of the wall. This effect is suppressed in multi wall nanotube. However, the diffraction of the radiation in multilayer wall system can not be neglected. 


\section{Carbon nanotube layers}

At present many techniques may produce layers of oriented nanotubes. Mostly aligned multiwall carbon nanotubes have been synthesized using zeolite-supported catalysts [54], lithographyc techniques [55, 56], and mixtures of ferrocene and fullerene $C_{60}$ or hydrocarbons compounds $[57,58,59]$. The nanotube structure and the orientation character change following the synthesis conditions. It should be underlined here that, nanotubes composed by one layer are often bent and this contributes to the no ideality of the film structure. A simple explanation of the formation of the nanotube structures is illustrated in Fig. 11.

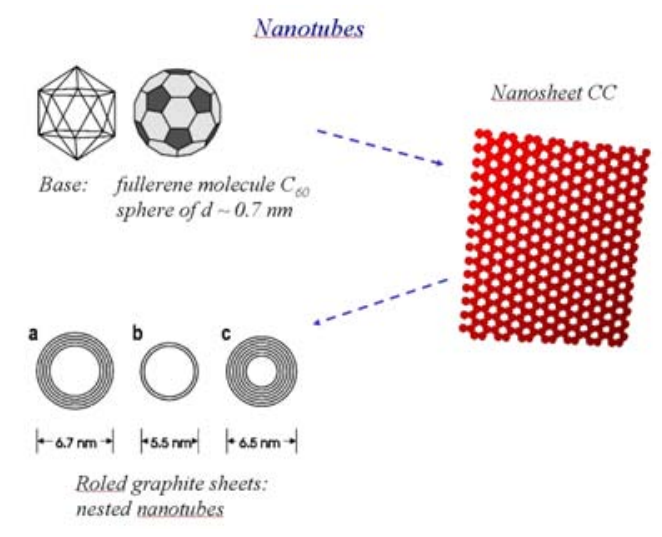

Figure 11: Evaluation of a carbon nanotube structure.

In our case a film of vertically aligned nanotubes on a $\mathrm{Si}$ substrate was prepared by thermal chemical vapor deposition (CVD) of ferrocene/fullerene mixture. The horizontal CVD apparatus consists of a stainless steel gas flow reactor of $1 \mathrm{~m}$ length and $3.4 \mathrm{~cm}$ diameter and a tubular furnace with a heating length of $30 \mathrm{~cm}$. The reactor is fitted with a removable quartz tube $3 \mathrm{~cm}$ in diameter. A ceramic boat with a mixture of fullerene $\mathrm{C}_{60}$ and ferrocene $\mathrm{Fe}\left(\mathrm{C}_{5} \mathrm{H}_{5}\right)_{2}$ taken in the ratio of (1:1) was placed inside the quartz tube under the Si plate of size $4 \times 10 \mathrm{~mm}^{2}$. The pyrolysis was performed at $950^{\circ} \mathrm{C}$ and atmospheric pressure in an argon flow (3 1/min).

The material produced was characterized by a scanning electron microscope (SEM) with a resolution of $\sim 10 \mathrm{~nm}$. Fig. 12 shows a SEM image of aligned carbon nanotube bundles deposited perpendicularly to the substrate surface. The mean length of nanotubes coincides with the thickness of a layer and is about $10 \mu \mathrm{m}$. The bundles are rather closely packed in the film and their density was estimated about 1000 pieces per $\mu \mathrm{m}^{2}$. For a second sample the layers of the oriented nanotubes were destroyed by pressing nanotubes onto a substrate surface. Due to the fact that nanotubes in the layer were quite long and defective, in the second investigated sample, nanotubes were mainly parallel to the layer surface.

As the TEM images show, multilayered carbon nanotubes, characterized by external diameter of $100 \div 200 \AA$, and diameter of an internal cavity of $50 \div 70$ Amay support the

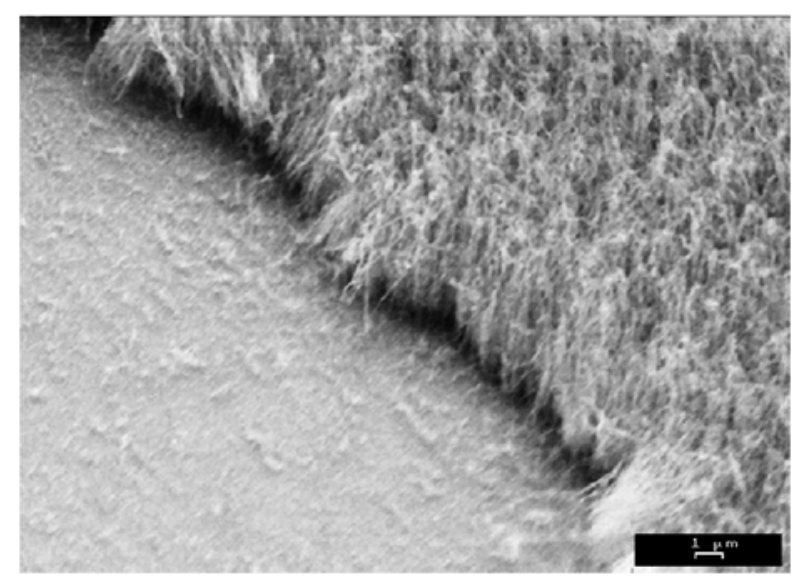

Figure 12: SEM image of a forest of the alligned carbon nanotubes.

growth of film structures that range from 5 up to 25 layers. Defects and bends of nanotubes are formed during growth, at destruction of a film or during the preparation of a sample for electron-microscopic measurements. In each layers the nanotubes are linked enough to form a sheaf of hundreds separate nanotubes, whose internal cavity is filled in part by metal iron.

From the SEM analysis we observed that nanotubes are most ordered in an internal part of a film. In the beginning of the growth process the silicon surface with deposited iron particles acts as the catalytic surface capable to support the alignment of carbon nanotubes. Then, a simultaneous growth does not allow tubes to be significantly bent. The external area of a film with thickness of $0.1 \div 0.2 \mu \mathrm{m}$ is probably the most disordered region due to the final contribution of the growing process, e.g., when the fullerene concentration is low.

\section{$X$-ray emission measurements and discussion}

The angular dependence of the x-ray fluorescence from nanotube films was measured using a laboratory spectrometer. Experimental layout was placed into the spectrometer chamber operating in a vacuum of $2 \cdot 10^{-6}$ Torr. The sample $8 \mathrm{~mm}$ long and about $2 \mathrm{~mm}$ wide was placed on the rotatable axis providing an angular range $\alpha$, between the sample surface and the optical axis of spectrometer from $60^{\circ}$ to $100^{\circ}$. The position of the copper anode of the $\mathrm{x}$-ray tube and the entrance slit was fixed. The angle $\beta$ between the radiation source (the anode surface is about $6 \mathrm{~mm}$ wide) and the rotating axis was $20^{\circ}$. A gas proportional counter with methane pressure of $0.2 \mathrm{~atm}$ was placed beyond the slit. A counter window was made from polypropylene film of $1 \mu \mathrm{m}$ thickness. At anode voltage of $2.3 \mathrm{kV}$ and current of $100 \mathrm{~mA}$ the maximal counter charge was $\sim 3000 \mathrm{im}$ pulses/sec. The total intensity at the maximum accounted for $\sim 60000$ impulses.

$\mathrm{X}$-ray fluorescence of a carbon atom does no show any angular dependence due to the localization and symmetry 
of the inner 1s-level. However, in crystals the probability of radiation emission is determined by the distribution of electrons in the valence band and may have a complex angular dependence. For example, in the emission x-ray spectra of graphite, the contribution of $\pi$ and $\sigma$ components is varied depending on angular orientation of the detector [60]. The shape of the $\mathrm{C} \mathrm{K}_{\alpha}$-spectrum of graphite is continuously changed with a take-off angle of radiation and an integral intensity of a spectrum almost constant.

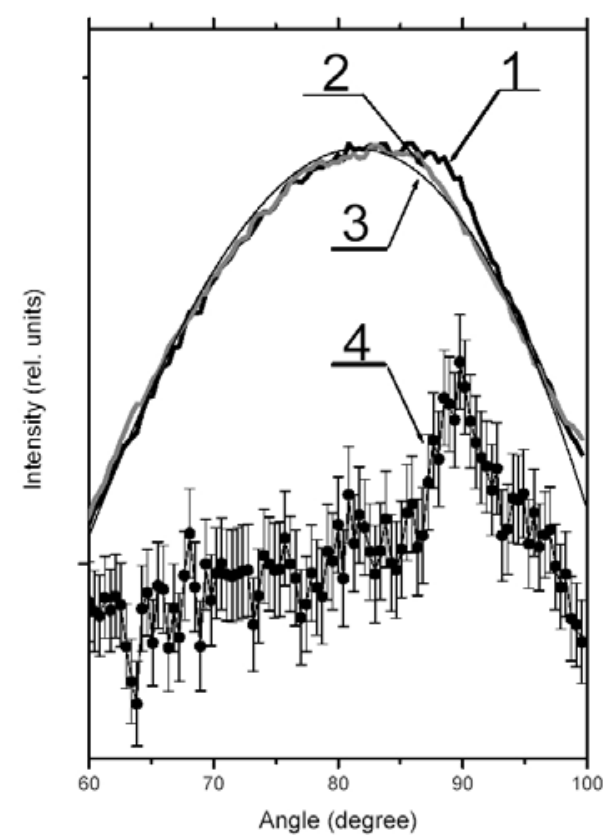

Figure 13: Angular dependence of X-ray emission from the aligned and chaotic nanotube arrangement in the carbon layer.

To detect effects due to channeling in carbon nanotubes the X-ray fluorescence from aligned nanotube film is compared to the emission of disordered nanotube film. The vertical orientation of carbon nanotubes in the second sample was destroyed by applying a pressure to the film. Indeed, after the treatment SEM analysis has shown an orientation almost parallel to the film surface. The angular dependence of the intensity of the x-ray fluorescence measured for both nanotube samples, aligned and disordered (curves 1 and 2, respectively) are compared in Fig.13. Intensity is normalized to the maximum at about $85^{\circ}$. All curves show a similar angular dependence increasing at angles from $60^{\circ}$ up to $85^{\circ}$. The sample of aligned carbon nanotubes is characterized by additional small feature around $90^{\circ}$. For the disordered film the angular dependence of the X-ray fluorescence intensity can be described with the geometrical parameters of the incoming beam and the emitted fluorescent beams. The measured intensity fits the following expression

$$
I=I_{0} \sin \alpha \cos \left(\alpha-20^{\circ}\right) \exp \left(\frac{-\eta \cos \left(\alpha-20^{\circ}\right)}{\sin \alpha}\right),
$$

where $I_{0}$ is the initial radiation intensity, $\eta$ is the cumulative factor included the absorption and the density of the sample. The formula takes into account the change of the measured area with an angle $\alpha$ and the absorption of the incident radiation and fluorescence when the sample thickness is larger than the characteristic absorption depth of the exciting radiation. The behaviour (22) is represented by curve 3 in Fig.13. The angular behaviour of the radiation intensity is a strong function of $\eta$ and using this parameter to fit curve 3 , we obtain a maximum at $82^{\circ}$.

The experimental angular dependence of the fluorescence emission is in good agreement with simulations and doesn't show features in the angular range near to the normal. The excitation of carbon x-ray fluorescence in nanotubes occurs through all the depth of a layer. However, the contributions of the radiation emitted by the deeper layers tend to decrease. On the other hand, the absorption of $\mathrm{C}$ $\mathrm{K}_{\alpha}$-radiation in a carbon layer is negligible. Therefore the contribution to the spectrum is given by the entire sample.

The difference between curves 1 and 2, multiplied by five is represented by the curve 4 in Fig.13. The intensity of this curve is estimated to be about $0.4 \%$ of the total $\mathrm{x}$ ray fluorescence intensity. Despite the large signal/noise ratio curve 4 exhibits a clear maximum at $90^{\circ}$ with a width of about $6^{\circ}$. This feature can be interpreted as due to the interaction with carbon nanotubes oriented in the direction of the radiation. The width is then determined by the deviation of carbon nanotubes from the ideal vertical alignment. X-ray fluorescence from carbon nanotubes significantly misaligned, which mainly occurs near to the sample surface, contribute to the diffuse halo. The complex form of curve 4 is actually determined by a combination of different phenomena of dispersion of the radiation from oriented nanotubes. We shall try to evaluate these contribution from the major factors of dispersion in the total angular distribution of the radiation behind the investigated structure.

Let us suppose that the angular distribution of the x-ray fluorescence from carbon nanotubes is isotropic so that we may estimate the mean angular deviation $\langle\Delta \theta\rangle$ of the x-ray fluorescence after traversing of a carbon layer. It may be calculated considering the complex mechanism of the scattering in the inner cavity and from the walls of nanotubes $\langle\Delta \theta\rangle_{\text {chan }}$ as well as by the diffuse scattering occurring in the space between separate nanotubes $\langle\Delta \theta\rangle_{\text {diff }}$. Hence, we can determine that

$$
\langle\Delta \theta\rangle=\langle\Delta \theta\rangle_{\text {diff }}+\langle\Delta \theta\rangle_{\text {chan }}
$$

The first term of the Eq.(23) depends by the texture of the nanotube's forest and by the mean distance between separate nanotubes or bundles of nanotubes tight attached to each other in the forest, i.e. $\langle\Delta \theta\rangle_{\text {diff }}=\langle\Delta \theta\rangle_{\text {text }}+$ $\langle\Delta \theta\rangle_{\text {pass }}$. The latter can be easily estimated from geomet- 
rical parameters while the microscopic pictures of the samples allow texture parameters to be defined. The analysis shows that for a layer thickness of $10 \mu \mathrm{m}$, the mean distance between separate nanotubes or bundles of nanotubes is about $100 \AA$. Moreover, we can evaluate also the mean angular deviation in the angular orientation of the forest of nanotubes of about $20^{\circ} \div 30^{\circ}$. Thus, for our samples the term $\langle\Delta \theta\rangle_{\text {pass }}$ corresponding to the straight ray propagation (without reflection "on a gleam", i.e., free propagation in a space between nanotubes) between the nanobundles in the carbon layer, is a few mrads (less than $1^{0}$ ) order from below. This value is lower than the angular divergence of an X-ray beam channeling in a nanotube. However, this factor has a small influence, i.e. the density of rays following a "straight propagation" is quite small. It is also reasonable, that the angular width of the dispersion due to the nanolayer texture $\langle\Delta \theta\rangle_{\text {text }}$ can be estimated with the same method, e.g., analyzing the layer micropicture and the angular orientational disorder of nanotubes within the layer. Disorder may be estimated by the mean deviation value of the angular orientation of nanotubes that is about $20^{\circ} \div 30^{\circ}$.

The second term of the expression (23) is the term that describe the radiation scattering inside the oriented channels of the layer. Following the definition of the scattering in channeling phenomena (see [61]), with a simple model we can write the following equation for the average angle of scattering in a nanotube $\left(e=\hbar=c=m_{0}=1\right)$

$$
\langle\Delta \theta\rangle_{\text {chan }} \sim \frac{1}{r_{0} N_{0}} \int_{\omega_{\min }}^{\omega_{\max }} d \omega \frac{N_{p h}(\omega)}{\omega} \int_{0}^{r_{0}} d x \omega_{0}(x)
$$

where $r_{0}$ is an internal radius of the "hollow" part of nanotube, $\omega$ and $N_{p h}(\omega)$ correspond to the emitted photon energy and the number of photons, $\omega_{0}(x)=\sqrt{4 \pi \rho(x)}$ is the energy of nanotube plasmon with a density of $\rho(x)=$ $\frac{e^{2}}{m_{0}} N_{e}(x)[49,50]$. From the expression $(24)$ we can estimate that $\langle\Delta \theta\rangle_{\text {chan }} \leq \omega_{0 \max } r_{0} / 10 \approx 6^{\circ} \div 10^{\circ}$ at the given values of $\omega_{0 \max } \simeq 100 \div 150 \mathrm{eV}$ and $r_{0} \simeq 20 \div 30 \AA$, that is in good agreement with the experimental data. Actually small angles of dispersion are affected by the interaction between x-ray quanta and electrons of the nanostructured media, which in nanotubes form axially symmetrical channel with smoothly varying density of the electronic cloud. Moreover it is also necessary to take into account the tunnel effect of quantum transition between channels in film formed by multiwall nanotubes. In the investigated samples the walls thickness has a range $\Delta d \sim 25 \div 65 \AA$ of the order or less than the transverse wavelength of the radiation $\lambda_{\perp} \sim 50 \div 100 \AA$. Therefore, the probability of tunneling into the neighbour channels, $\propto \exp \left(-\Delta d / \lambda_{\perp}\right)$, is large enough. However, the process of tunneling is accompanied also by small-angle diffraction. The presence of a set of ordered walls introduces diffraction dispersion of x-ray and the first maximum mainly determined by diffraction, is observed at angles close to the angles of channeling [62].

A significant portion of the radiation mainly scattered at the condition of channeling by the hollow part of the nanotubes, is not guided due to the distributed disorder of the nanosystem orientation. In other words, a large angular width of the orientation distribution of the nanotubes leads to a fast dechanneling of the radiation due to the increase of the "reflection angles" from nanotube walls. Then the scattering of this dechanneled fraction of the beam is dominated by diffusion phenomena in the nanotube forest and explains also the weak peak in the angular distribution of the emerging radiation.

Modern technological manufacture processes addressed in the last years the scientific relevance of systems formed by layers of focusing nanotubes. The question about the possible use of hollow channels in nanostructures, discussed theoretically in the last years appears now feasible. However, the quality of these films is still far from ideal in order to be used as devices with defined crystal directions. Nevertheless, it is now possible start preliminary investigations about the structural effects of radiation dispersion in carbon nanotubes. In these systems the observed effects on the micron scale are due to the small sizes of the channels.

\section{ACKNOWLEDGEMENTS}

I would like to thank all our collaboration for studying coherent phenomena of radiation scattering in microand nanostructures of different periodicity, namely Prof. L. Palumbo and his group by SPARC for their interest and help in organizing channeling projects within the FEL programs of Frascati, Prof. N.F. Shul'ga and group of Kharkov PhTI for theoretical analysis of relativistic electron scattering in crystals and proposal for new beamline construction, Prof. M.A. Kumakhov and scientists of IRO for their valuable contribution to studying of X-ray transmition by capillary systems, Profs. W. Greiner, A. Soloviev, H. Backe and Beam Test Facility group of LNF for their interest to study channeling undulator features for relativistic positrons. I am sincerely appreciated many fruitful discussions with Profs. X. Artru, M.A. Kumakhov, A Okotrub, and N. Nasonov, Drs. N. Zhevago and K. Ispirian and their promising proposals. I am particularly grateful to Profs. Herbert Überall, Giordano Diambrini Palazzi and Dr. Gianpaolo Murtas for the invaluable historical and scientific information on pioneering studies of coherent radiation in crystals. At last I would like to thank the participants of the International Workshop on Charged and Neutral Particles Channeling Phenomena "Channeling 2004" for the extremely high-level contributions.

\section{REFERENCES}

[1] B. Ferretti, Nuovo Cimento 7 (1950) 118.

[2] M.L. Ter-Mikaelian, Zh. Eksp. Teor. Fiz. 25 (1953) 296.

[3] F.J. Dyson, and H. Überall, Phys. Rev. 99 (1955) 604.

[4] E.L. Feinberg and I. Pomeranchuk, Nuovo Cimento Suppl. 3-X (1956) 652.

[5] H. Überall, Phys. Rev. 103 (1956) 1055. 
[6] H. Überall, Phys. Rev. 107 (1957) 223.

[7] R. Frisch, and D. H. Olson, Phys. Rev. Lett. 3 (1959) 141.

[8] W.R.H. Panofsky and A.N. Saxena, Phys. Rev. Lett. 2 (1959) 219.

[9] G. Diambrini Palazzi, Rev. Mod. Phys. 40(3) (1968) 611.

[10] G. Bologna, G. Diambrini Palazzi, and G. P. Murtas, Phys. Rev. Lett. 4 (1960) 134.

[11] G. Bologna, G. Diambrini Palazzi, and G. P. Murtas, Phys. Rev. Lett. 4 (1960) 572.

[12] G. Diambrini Palazzi, Proc. Conf. Photon Interactions in the BeV Energy Range, Cambridge, Mass., VI (1963) 4.

[13] G. Barbiellini, G. Bologna, G. Diambrini Palazzi, and G. P. Murtas, Phys. Rev. Lett. 8 (1962) 112.

[14] G. Barbiellini, G. Bologna, G. Diambrini Palazzi, and G. P. Murtas, Phys. Rev. Lett. 8 (1962) 454.

[15] G. Barbiellini, G. Bologna, G. Diambrini Palazzi, and G. P. Murtas, Phys. Rev. Lett. 9 (1962) 396.

[16] G. Barbiellini, G. Bologna, G. Diambrini Palazzi, and G. P Murtas, Nuovo Cimento 28 (1963) 436.

[17] G. Bologna, G. Lutz, H. D. Schultz, U. Timm, and W. Zimmermann, Nuovo Cimento 42 (1966) 844.

[18] U. Timm, Fortschritte Der Physik 17 (1969) 12.

[19] L. Criegee, G. Lutz, H. D. Schultz, U. Timm, and W. Zimmermann, Nuovo Cimento 16 (1966) 1031.

[20] S. Kato, T. Kifrne, Y. Kimura, M. Kobayashi, K. Kondo, T. Nishikawa, H. Sasaki, K. Takanratsu, S. Kikuta, and K. Kohra, J. Phys. Soc. Japan 20 (1965) 303.

[21] D. Alesini et al. "Status of the SPARC Project", presented at PAC2005 16-20/5/2005, Knoxville, Tennessee, USA.

[22] http://www.sparx.it/

[23] http://ilil.ipcf.cnr.it/plasmonx

[24] Coherent Radiation Sources (A. W. Saenz and H. Überall, Eds), Springer, Berlin, 1985.

[25] Proceedings of "Radiation from Relativistic Electrons in Periodic Structures", Nucl. Instr. Meth. B145(1,2) (1998).

[26] M.Kh. Khokonov, and R.A. Carrigan, Jr., Nucl. Instr. Meth. B145(1,2) (1998)133.

[27] N.F. Shul'ga, private communication.

[28] Radiation of Channeling Particles, M.A. Kumakhov,Ed., Energoatomizdat, Moscow, 1986.

[29] "Kumakhov Optics and Application", Kumakhov M.A., Ed., Proc. of SPIE 4155 (2000).

[30] S.B. Dabagov, Research Report of FIROS, 1992.

[31] S.B. Dabagov, and M.A. Kumakhov, Proc. of SPIE 2515 (1995) 124.

[32] S.B. Dabagov, M.A. Kumakhov, and S.V. Nikitin, Phys. Lett.A203 (1995) 279.

[33] G. Cappuccio, S.B. Dabagov, A. Pifferi, and C. Gramaccioni, Appl. Phys. Lett. 78 (2001) 2822.

[34] 17th International Congress on X-Ray Optics and Microanalysis, Chamonix, France (September 22-26, 2003).

[35] S.B. Dabagov, M.A. Kumakhov, S.V. Nikitina, et al., J. Synchrotron Rad. 2 (1995) 132.
[36] N. Artemiev, A. Artemiev, V. Kohn, and N. Smolyakov, Phys. Scripta 57 (1998) 228.

[37] S.B. Dabagov, and A. Marcelli, Appl. Opt. 38, 7494 (1999).

[38] S.V. Kukhlevsky, F. Flora, A. Marinai, et al., Nucl. Instr. Meth. B168, 276 (2000).

[39] S.B. Dabagov, V.A. Murashova, N.L. Svyatoslavsky, et al., Proc. SPIE. 3444, 486 (1998).

[40] S.B. Dabagov, A. Marcelli, V.A. Murashova, et al., Appl. Opt. 39 (2000) 3338.

[41] 5. S.B. Dabagov, A. Marcelli, G. Cappuccio, and E. Burattini, Nucl. Instr. Meth. B187(2) (2002) 169.

[42] S.B. Dabagov, A. Marcelli, V.A. Murashova, et al., Proc. SPIE 4138 (2000) 79.

[43] Chien Liu, and J.A. Golovchenko, Phys. Rev. Lett., 79 (1997) 788.

[44] Yu.M. Alexandrov, S.B. Dabagov, M.A. Kumakhov, et al., Nucl. Instr. Meth. B134 (1998) 174.

[45] S. Iijima, Nature 354 (1991) 56.

[46] Physical Properties of Carbon Nanotubes, R. Saito, G. Dresselhaus and M. S. Dresselhaus,Eds., Imperial College Press, London, 1998.

[47] L. Langer, V. Bayot, E. Grivei et al. Phys. Rev. Lett. 76 (1996) 479.

[48] V.V. Klimov, and V.S. Letokhov, Phys. Lett.A222(1996) 424.

[49] G.V. Dedkov, Nucl. Instr. Meth. B143(1998)584.

[50] N.K. Zhevago, and V.I. Glebov, Phys. Lett. A250(1998) 360.

[51] E. Burattini, S.B. Dabagov and F. Monti, Nuovo Cimento 117B (2002) 769.

[52] S.B. Dabagov, Physics Uspekhi 46(10) (2003)1053.

[53] D. Gemmell, Rev. Mod. Phys. 46(1974) 129.

[54] W.Z. Li, S.S. Xie, L.X. Qian et al. Science 274 (1996) 1701.

[55] J.I. Sohn, and S. Lee, Appl. Phys. A74 (2002) 287.

[56] M. Terrones, N. Grobert, J. P. Zhang et al. Chem. Phys. Letters285 (1998) 299.

[57] M. Mayne, N. Grobert, M. Terrones et al. Chem. Phys. Letters338 (2001) 101

[58] N. Grobert, W.K. Hsu, Y.Q. Zhu et al. Appl. Phys. Lett.75 (1999) 3363.

[59] B.C. Satishkumar, A. Govindaraj, and C.R. Rao, Chem. Phys. Letters307 (1999) 158.

[60] Chr. Beyreuther, R. Hierl, and G. Wiech, Berlichte der Bunsen-Gesselschaft für Phys. Chem.79 (1975) 1081.

[61] S.B. Dabagov, Rad. Eff. Def. Solids 25 (1993) 103.

[62] A.V. Okotrub, S.B. Dabagov, A.G. Kudashev et al. ZhETF Letters 81(1) (2005) 34. 